\title{
Tetrel bond: dipyridyl methylene as a donor site
}

\author{
M. Calabrese, A. Daolio, A. Pizzi, G. Resnati \\ NFMLab - Department of Chemistry, Materials and Chemical Engineering “Giulio Natta”, Politecnico di Milano, Via L. Mancinelli \\ 7, I-20131 Milan, Italy \\ miriam.calabrese@polimi.it
}

$\sigma$-Hole interactions are a subclass of non-covalent interactions wherein an area of positive electrostatic potential on the extension of a covalent bond forms a net attractive interaction with an electron-rich site. The tetrel Bond (TtB) is a $\sigma$-hole interaction where the electrophilic atom is an element of Group 14 of the periodic table. The strong interest in this interaction is related to the pivotal role of carbon in organic and bio-chemistry [1].

However, the reduced polarizability of carbon makes this element the less prone in the Group to be involved in TtBs formation. In relation to our work on methonium derivatives as TtBs donors [2], we propose bis-pyridinium methylene salts as a second model system to study the geometric and energetic features of this force on $-\mathrm{CH}_{2}$ - derivatives. The obtained crystal structures present short and linear contacts between the methylene carbon and various nucleophiles; the formation of these interactions is interpreted as the result of the strong electron withdrawing effect of the positive nitrogen atoms directly bound to the carbon.

The crystal structures were studied with various tools to assess the nature of the contact, resulting in the unequivocal confirmation that the interactions presented should be considered TtBs, thus complementing our previous study on barbituric acid derivatives [3]. TtBs on these compounds are found to be robust enough to be able to drive the crystalline packing of specific adducts and build distinctive architectures.

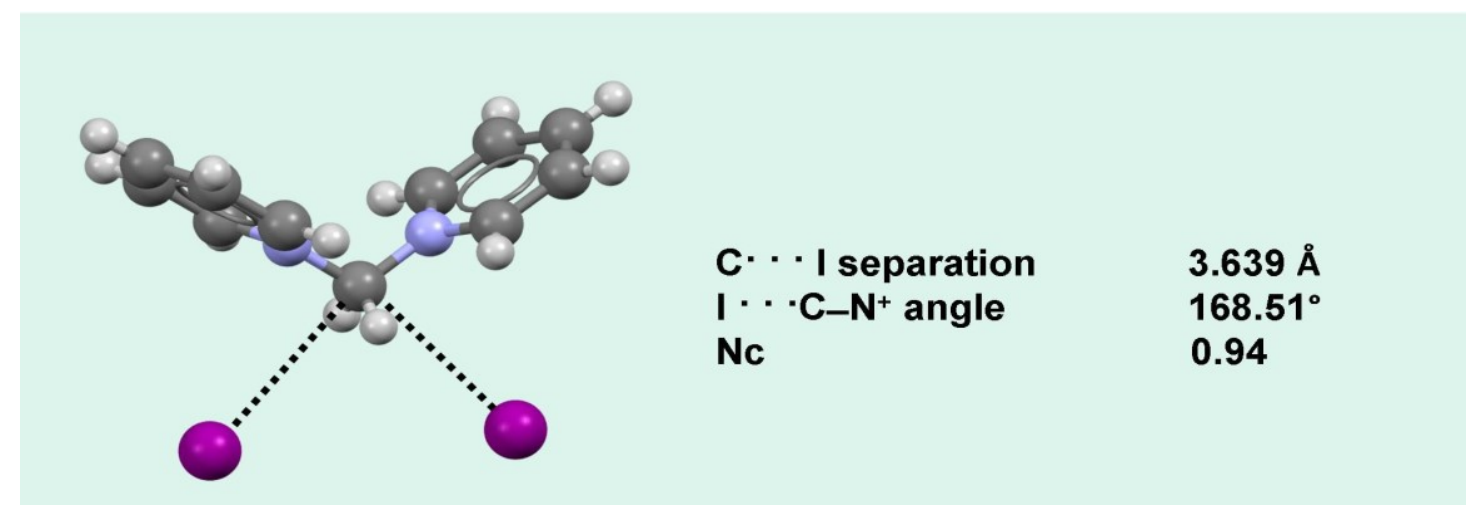

Figure 1. Ball and stick representation of one representative structure described in this work. TtBs are black dotted lines, geometrical features of the interaction are displayed close to the lines. Color code: grey carbon, whitish hydrogen, light blue nitrogen, purple iodine.

[1] Daolio, A. Scilabra, P., Terraneo, G. \& Resnati, G., C(sp $\left.{ }^{3}\right)$ atoms as tetrel bond donors: A crystallographic survey, Coord. Chem. Rev. (2020) 413: 213-265

[2] Daolio A., Wieduwilt E., Genoni A., Terraneo G., Resnati G., Manuscript in preparation

[3] Vijith K., Scilabra P., Politzer P., Terraneo G., Daolio A., Fernandez- Palacio F., Murray J. S., Resnati G., Tetrel and Pnictogen Bonds

[4] Complement Hydrogen and Halogen Bonds in Framing the Interactional Landscapeof Barbituric Acids, Cryst. Growth Des. 2021, 21, 1, 642-652

Keywords: $\sigma$-Hole, Non-covalent interaction, Tetrel bond 